\title{
Cooperative Interaction between the Alpha1-Adrenoceptors ( $\alpha$ 1-AR) and Transient Receptor Potential (TRP) Triggers a Proliferative Cell Signal in Prostate Cancer Cell Lines
}

Giorgio Santoni ${ }^{1 *}$, Maria Beatrice Morelli ${ }^{1,2}$, Consuelo Amantini ${ }^{3}$, Matteo Santoni ${ }^{4}$, Massimo Nabissi ${ }^{1}$, Claudio Cardinali ${ }^{1,4}$, Fabio Del Bello ${ }^{5}$, Alessandro Piergentili ${ }^{5}$ and Wilma Quaglia ${ }^{5}$

${ }^{1}$ School of Pharmacy, Experimental Medicine Section, University of Camerino, Camerino, Italy

${ }^{2}$ Department of Molecular Medicine, Sapienza University, Rome, Italy

${ }^{3}$ School of Biosciences and Veterinary Medicine, University of Camerino, Camerino, Italy

${ }^{4}$ Department of Medical Oncology, AOU Ospedali Riuniti, Polytechnic University of the Marche Region, Ancona, Italy

${ }^{5}$ School of Pharmacy, Medical Chemistry Unit, University of Camerino, Camerino, Italy

\begin{abstract}
Calcium ( $\mathrm{Ca} 2+)$ increases the proliferation of human advanced prostate cancer ( $\mathrm{PCa}$ ) cells but the ion channels involved are unknown. Santoni and Quaglia groups investigated the correlation between alpha1D-adrenergic receptor (a1D-AR) and the transient receptor potential vanilloid type 1 (TRPV1) ion channel expression in PCa cells. The a1D-AR and TRPV1 mRNAs are increased in PCa compared to BPH. a1D-AR and TRPV1 are co-expressed in PCa cells. Norepinephrine (NE) induced a1D-AR- and TRPV1-dependent protons release, Ca2+ flux in PC3 cells and activation of PLC, PKC and ERK path-ways that stimulated PC3 cell proliferation. Similarly, a role for TRPC6 or GPR55 in a1-AR-dependent proliferation of mesangial cells and PCa cells was reported.
\end{abstract}

Overall, a crosstalk between a1-AR and TRPs in PCa cells, involved in the control of cell proliferation has been demonstrated. These data strongly promote a putative novel pharmacological approach in the treatment of PCa by targeting both $\alpha 1-\mathrm{AR}$ and TRP channels.

Keywords: Prostate cancer; Transient receptor potential vanilloid type-1; Alpha1D-adrenergic receptors; Proliferation; Mitogen activated protein kinase

\section{Introduction}

Calcium is essential for enhancing the proliferation in prostate cancer (PCa) cells [1]. Although, several calcium channels have been identified, the molecular mechanisms leading to the activation of $\mathrm{Ca} 2+$ signaling pathways in PCa are partially unknown. The knowledge of the factors triggering PCa cells to increased cell proliferation is very important for the development of novel therapies preventing the growth and progression. Alpha1-adrenoceptors ( $\alpha 1$-ARs) respond to epinephrine (E) and norepinephrine (NE, also called noradrenaline) specific ligands in different target tissues. a1A-AR, a1B-AR and $\alpha 1 \mathrm{D}-$ ARs types are expressed in benign prostate hyperplasia (BHP), PCa, in the androgen-resistant PC3 and DU-145 and androgen-sensitive LNCap cell lines [2,3]. The al-ARs belong to the G-protein-coupled receptors (GPCRs). They are linked to the heterotrimeric $\mathrm{G}$ protein containing the Gaq/11/14/16 subunits that activates phospholipase $\mathrm{C}$ (PLC) and promotes the cleavage of inositol 4,5-bisphosphate (PIP2) to diacylglycerol (DAG) and inositol 1,4,5-triphosphate (IP3); DAG and IP3 then promote the activation of protein kinase C (PKC). Evidence suggests the al-ARs contribute in the catecholamines-dependent prostate cell growth [4]. In this view, the capability of NE to trigger the proliferation of PC-3 prostate cancer cells in a1D-AR-dependent manner has been reported [3].

\section{Functional interaction between the a1D-AR and TRPV1 ion} channels in PC3 cell line

Among the calcium channels regulating the $\mathrm{Ca} 2+$ homeostasis in PCa cells, members of transient receptor potential (TRP) ion channel family seem to play a major role, and has been hypothesized that abnormal Ca2+ signaling may be an essential step in the a1-ARmediated increased proliferation in PCa cells [2,5]. Previously, we have reported the role of a1D-AR in NE-induced proliferation of PC3 cells [2]. However at present, no data on the type of $\mathrm{Ca} 2+$ channels involved in PCa proliferation have been reported. The transient receptor potential vanilloid type-1 channel (TRPV1) is expressed in human normal prostate epithelial cells, $\mathrm{PCa}$ and in PC-3 and LNCaP cell lines. Its surface expression was up-regulated in $\mathrm{PCa}$, compared with $\mathrm{BPH}$ tissues, and TRPV1 channel expression correlates with increasing pathological grade [3]. Santoni and Quaglia groups demonstrated that in PCa cells a1D-AR and TRPV1 colocalized in the plasma membrane, perinuclear region and intracellular vesicles [3].

Moreover, in PCa intense functional interaction between the a $1 \mathrm{D}$ AR and TRPV1 receptors has been demonstrated. Cross-sensitisation between different members of TRP and GPCR families, such as the chemokine receptor, e.g., CCR1 in DRG neurons and HEK-293 cells respectively, has been reported [6,7]. a1D-AR displays higher affinity for endogenous catecholamines compared to $\alpha 1 \mathrm{~A}-$ and $\alpha 1 \mathrm{~B}-\mathrm{AR}$ subtypes. Santoni et al. demonstrated that binding of NE to a1D-AR sensitizes the TRPV1 channels in PC3 cells [2]. The trigger of PCa cells with NE resulted in protons release and $\mathrm{Ca} 2+$ flux, which was abrogated by both

*Corresponding author: Giorgio Santoni, PhD, School of Pharmacy, Experimental Medicine Section, University of Camerino, Via Madonna delle Carceri, Camerino-62032, Italy, Tel: +39 0737403312, Fax: +39 0737630618 E-mail: giorgio.santoni@unicam.it

Received September 03, 2015; Accepted November 04, 2015; Published November 11, 2015

Citation: Santoni G, Morelli MB, Amantini C, Santoni M, Nabissi M, et al. (2015) Cooperative Interaction between the Alpha1-Adrenoceptors ( $\alpha 1-A R$ ) and Transient Receptor Potential (TRP) Triggers a Proliferative Cell Signal in Prostate Cancer Cell Lines. J Genet Syndr Gene Ther 6: 275. doi:10.4172/2157-7412.1000275

Copyright: ( 2015 Santoni G, et al. This is an open-access article distributed under the terms of the Creative Commons Attribution License, which permits unrestricted use, distribution, and reproduction in any medium, provided the original author and source are credited. 
Citation: Santoni G, Morelli MB, Amantini C, Santoni M, Nabissi M, et al. (2015) Cooperative Interaction between the Alpha1-Adrenoceptors ( $\alpha 1-A R$ ) and Transient Receptor Potential (TRP) Triggers a Proliferative Cell Signal in Prostate Cancer Cell Lines. J Genet Syndr Gene Ther 6: 275. doi:10.4172/2157-7412.1000275

Page 2 of 3

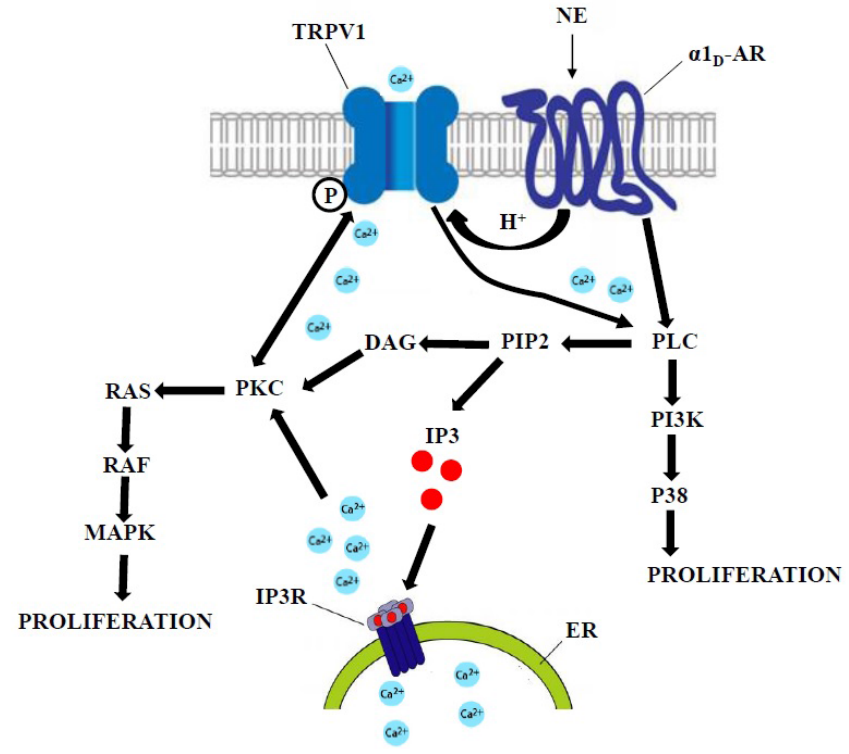

Figure 1: A simplified scheme of regulatory cross-talk between a1D-adrenergic receptor ( $\alpha 1 \mathrm{D}-\mathrm{AR})$ and Transient Receptor Potential Vanilloid 1 (TRPV1) that includes multiple parallel and converging downstream signal transduction pathways. DAG: diacylglycerol; IP3: inositol 1,4,5-trisphosphate; IP3R: IP3 receptor; MAPK: mitogen activated protein kinases; NE: norepinephrine; PI3K: phosphatidylinositol-4,5-bisphosphate 3-kinase; PIP2: phosphatidylinositol 4,5-bisphosphate; PKC: protein kinase C; PLC: phospholipase C.

the a1D-AR and TRPV1 antagonists, WS433 and capsazepine (CPZ). Furthermore, NE triggers a crosstalk between a1D-AR and TRPV1 receptors in PC3 cells through the PLC-PKC-ERK pathways. NE sensitizes the TRPV1 channels to activate the PLC pathway [3]. Ca2+ flow through the TRPV1 channel activates PLC, and the consequent PIP2 depletion promotes the TRPV1 sensitization in a PKC-dependent manner. In addition, the inhibition of a1D-AR and TRPV1 channels completely abrogated ERK $1 / 2$ phosphorylation, suggesting that the MAP kinases, ERK1 and ERK2 represent downstream components of NE-induced TRPV1/a1D-AR signaling pathway [3] (Figure 1). To further support these data, a complete abrogation of NE-induced increased PC3 cell proliferation was observed only in double-silenced a1D-AR/TRPV1 PC3 cells. Silencing of a1D-AR or TRPV1 gene or the use of $\alpha 1 \mathrm{D}-\mathrm{AR}$ and TRPV1 antagonists, alone, were unable to completely inhibit the NE-mediated effects, suggesting that in PC3 prostate cancer cells, these receptors play a cooperative functional role in mediating $\mathrm{Ca} 2+$ signals [3].

\section{TRPC6 channels are involved in a1-AR induced proliferation in human mesangial cell line}

Calcium influx by the stimulation of $\alpha 1-A R$ by TRPC ion channels is often mediated via ROCE or SOCE, rather than by voltage-gate Ca2+ channels. Similarly to prostate tissues, a1-AR is expressed in human mesangial cell line. In these cells, the $\alpha 1-\mathrm{AR}$ agonist phenylephrine $(\mathrm{PE})$ induced $\mathrm{Ca} 2+$ influx and release from intracellular $\mathrm{Ca} 2+$ stores. Blockage of TRPC6 with siRNA, anti-TRPC6 antibodies and a TRPC blocker attenuated the PE-induced $\mathrm{Ca} 2+$ increase in a phospholipase $\mathrm{C}$ dependent-manner [8]. In addition, $\mathrm{PE}$ induced a $\mathrm{Ca} 2+$ increase even when the intracellular $\mathrm{Ca} 2+$ stores were already depleted; this effect was mimicked by a DAG analog suggesting that, upon a1-AR stimulation, the TRPC 6 channel mediates Ca2+ influx. This concept is supported by recent studies that snapin, a regulator of receptor signaling, augments the a1-AR -induced Ca2+ influx through TRPC6. However, the possibility that other TRPC channels, expressed in mesangial cells (MCs) are involved in a1-AR agonist-induced $\mathrm{Ca} 2+$ influx could not to be excluded, given that TRPC6 can form heteromeric channel complexes with other TRPC channels. This channel also contributes to the PE-induced MCs proliferation, and this effect in various cell types is mediated via the mitogen-activated protein kinase (MAPK) family, including ERK, c-Jun N-terminal kinases (JNKs) and p38 kinases; however, the underlying mechanism is not completely clear [8]. The al-AR activation-induced proliferation of MCs is associated with ERK1/2 phosphorylation, and TRPC6 contributes to ERK signaling because the application of TRPC6 siRNA, the TRPC blocker, or chelation of extracellular $\mathrm{Ca} 2+$ attenuated a1-AR activation-induced ERK1/2 phosphorylation. These data have been also supported by the evidence that TRPC6 induces ERK activation in other cell types such as podocytes, neurons and islet cells. In addition in MCs, ERK1/2 may directly activate TRPC6 by phosphorylation at Ser-281. Several other signaling pathways were shown to be regulated downstream of TRPC6 such as the calcineurin-NFAT (nuclear 5 factor of activated T-cells) and RhoA pathways. Overall, in human MCs, TRPC6 channels are involved in a1-AR activation-induced $\mathrm{Ca} 2+$ entry, which mediates proliferation via ERK signaling [8]. Therefore, the possibility that TRPC6 mediates MC proliferation in normal and neoplastic tissues in response to a1AR activation via other signaling pathway could not be excluded and should be further evaluated.

\section{a1-ARs and G protein coupled receptor 55 co-localize in PCa cells}

Recently, a1-ARs and G Protein Coupled Receptor 55 (GPR55) belonging to (GPCR) family have been demonstrated to play a crucial role in regulating prostate function [9]. By confocal microscopy, fluorescent ligands showed heterogeneous expression of both a1-ARs and GPR55 in PC3 and LNCaP cells; some PCa cells express both a1ARs and GPR55 in relatively equal numbers indicating a degree of colocalization. Co-localization of fluorescent ligand binding was stronger in LNCaP respect to PC-3 cells. The presence of subtype-rich cells with a degree of co-localization between a1-ARs and GPR55 indicates a possible dimerization or a converging intracellular signaling process [10]. The pre-requisite of this event is that the two receptors should be in close proximity, influencing their ability to transduce signals. In addition, the observation that PC3 cells, high in GPR55 expression, have an high proliferation rate when returned to drug-free media. These results strongly support the hypothesis that in clinical setting, removal of a1-antagonism and the resultant stimulation of a1-AR may allow the formation of the lysophospholipid, lysophosphatidylinositol (LPI), an endogenous ligand for GPR55, synthesized intracellularly from membrane phosphoinositols by phospholipase A2 (PLA2), pumped out of the cells by the ATP-binding cassette transporter ABCC1/MRP1, that stimulate GPR55-mediated increased proliferation. LPI is able to induce calcium mobilization and activation of Akt and extracellular signal-regulated kinase (ERK) $1 / 2$ in cancer cells and pharmacological blockade of GPR55 using specific small interfering RNA strongly inhibits proliferation and anchorage-independent growth.

In addition, the a1-ARs/GPR55 ratio may serve as a useful biomarker or predictor of metastatic PCa [9]. Thus, the use of stable, non-toxic fluorescent ligands for these receptors permits to detect the 'hot' cells in a biopsy, by uropathologists to monitor the efficacy of anticancer therapy which the individual is undergoing and gauge the potential/progression to aggressive PCa. 
Citation: Santoni G, Morelli MB, Amantini C, Santoni M, Nabissi M, et al. (2015) Cooperative Interaction between the Alpha1-Adrenoceptors ( $\alpha 1-A R$ ) and Transient Receptor Potential (TRP) Triggers a Proliferative Cell Signal in Prostate Cancer Cell Lines. J Genet Syndr Gene Ther 6: 275. doi:10.4172/2157-7412.1000275

Page 3 of 3

\section{Conclusion}

These studies suggested a direct interaction between TRP, endogenous cannabinoid and non-cannabinoid receptors with different members of GPCRs family (e.g., a1-AR, CCR1, GPR55) through co-localization/dimerization, thus providing the rationale for further studies on the crosstalk between different receptors [10]. These results open a new avenue of research oriented on delineating direct physical and functional interactions between the cannabinoid receptors (e.g., TRPV1, GPR55) and the adrenergic system. Therefore, in the light of our and others findings, it is clear the needless of a reevaluation of the interactions between adrenergic and TRP and non-CB receptor systems in cells and tissues that co-express both, using higher resolution techniques with fluorescent ligands. The recent observation that GPR55 and TRP (TRPV1, TRPC6) are expressed in PCa cell line, suggests that some of the anti-tumor effects of al-AR antagonist may be the result of an indirect action on these receptors. Since TRP receptors have emerged as modulator of oncogenesis, autocrine signaling system warrants focused research to develop novel therapeutics targeting the protein or its ligand for the purposes of inhibiting cancer growth and limit the risk of metastases [3]. In this regard, the search of new strategies to target ion channels for therapeutic applications has become of increasing interest. For example, the TRPV1 channel that is, virtually not expressed in normal human prostate tissue, but overexpressed in high invasive prostate cancers could represent a novel tumor marker with diagnostic and therapeutic potential.

Overall, these findings strongly support for the introduction of novel pharmacological approaches in the care of advanced prostate cancer, such as the antibody-driven cancer therapy with the use of bispecific antibodies, showing high selectivity to target cells or the use of non-toxic fluorescent ligands able to directly contact specific receptors expressed in cancer cells and consequently to abrogate the proliferative cancer signals.

\section{Financial Support}

This study was supported by AIRC IG 2014-16, cod. 15821.

\section{References}

1. Flourakis M, Prevarskaya N (2009) Insights into Ca2+ homeostasis of advanced prostate cancer cells. Biochim Biophys Acta 1793: 1105-1109.

2. Quaglia W, Santoni G, Pigini M, Piergentili A, Gentili F, et al. (2005) Structure-activity relationships in 1,4-benzodioxan-related compounds. 8 (1) $\{2$-[2-(4-chlorobenzyloxy)phenoxy]ethyl\}-[2-(2,6-dimethoxyphenoxy)ethyl] amine (clopenphendioxan) as a tool to highlight the involvement of alpha1Dand alpha1B-adrenoreceptor subtypes in the regulation of human PC3 prostate cancer cell apoptosis and proliferation. J Med Chem 48: 7750-7763.

3. Morelli MB, Amantini C, Nabissi M, Liberati S, Cardinali C, et al. (2014) Crosstalk between alpha1D-adrenoceptors and transient receptor potential vanilloid type 1 triggers prostate cancer cell proliferation. BMC Cancer 14: 921.

4. Hennenberg M, Stief CG, Gratzke C (2014) Prostatic a1-adrenoceptors: new concepts of function, regulation, and intracellular signaling. Neurourol Urodyn 33: $1074-1085$.

5. Thebault S, Roudbaraki M, Sydorenko V, Shuba Y, Lemonnier L, et al. (2003) Alpha1-adrenergic receptors activate $\mathrm{Ca}(2+)$-permeable cationic channels in prostate cancer epithelial cells. J Clin Invest 111: 1691-1701.

6. Zhang N, Inan S, Cowan A, Sun R, Wang JM, et al. (2005) A proinflammatory chemokine, CCL3, sensitizes the heat- and capsaicin-gated ion channel TRPV1. Proc Natl Acad Sci U S A 102: 4536-4541.

7. Thebault S, Flourakis M, Vanoverberghe K, Vandermoere F, Roudbaraki M, et al. (2006) Differential role of transient receptor potential channels in Ca2+ entry and proliferation of prostate cancer epithelial cells. Cancer Res 66: 2038-2047.

8. Kong F, Ma L, Meng K, Zhang L, Zhang R, et al. (2015) Alpha1-adrenergic receptor activation stimulates calcium entry and proliferation via TRPC6 channels in cultured human mesangial cells. Cell Physiol Biochem 36: 19281938.

9. Patil KC, McPherson L, Daly CJ (2015) Co-localization of alpha1-adrenoceptors and GPR55: a novel prostate cancer paradigm? Pharmacology and Pharmacy 6: $212-220$.

10. Terrillon S, Bouvier M (2004) Roles of G-protein-coupled receptor dimerization EMBO Rep 5: 30-34. 